\title{
SOME FACTORS INVOLVED IN THE STIMULUS
}

\section{CONTROL OF OPERANT BEHAVIOR}

\author{
W.H. MORSE AND B.F. SKINNER
}

Harvard University

The stimuli which are present when an operant is reinforced modify the subsequent frequency of emission of the response. For example, if a hungry pigeon is reinforced with food when it pecks a translucent key upon which a monochromatic light is projected, it will subsequently peck most rapidly when the light is of the same wavelength. Guttman(3) has reported that a difference of 2 millimicrons can produce a lower rate. As the difference increases the rate falls in a "generalization gradient." If responses are reinforced at one wavelength and extinguished at all others, the gradient is sharpened. These facts are now well known, but the significance of the relevant conditions has not been fully analyzed.

When a response is reinforced in the presence of a stimulus, the stimulus $\left(\mathrm{S}^{D}\right)$, the response $(\underline{R})$, and the reinforcement ( $\mathrm{rft}$ ) occur in close temporal proximity, as the result of certain arbitrary arrangements. At first, $\mathrm{SD}$ usually has no demonstrable control over the response; it does not elicit the response as in a reflex and is chosen from many possible stimuli. The relation between $R$ and $\mathrm{rft}$ is arranged by the reinforcing circuit. Once these two connections have been established, there is a fairly close temporal contiguity between $\mathrm{SD}$ and $\mathrm{rft}$. But which of these relations is responsible for the stimulus-generalization gradient (with or without differential reinforcement)? In particular, is the role of the response in bringing $\mathrm{S}^{\mathrm{D}}$ and $\mathrm{rft}$ close together essential to the development of stimulus control, or is the temporal association between $\mathrm{SD}$ and $\mathrm{rft}$ enough to make any $\underline{\mathrm{R}}$ which produces $\underline{\mathrm{rtt}}$ more frequent in the presence of $\underline{\mathrm{SD}}$.

This point has been the subject of several experiments. K. Walker (5) has shown that a discriminative stimulus developed in connection with one operant has an appropriate effect upon another. The introduction of a tone which had been the discriminative stimulus for a running response facilitated lever-pressing. Estes (1) reinforced pressing a lever on a fixed-interval schedule, then removed the lever and repeatedly presented a stimulus for 60 seconds before the "free" presentation of food. When the levers were replaced and the response extinguished, a higher rate was observed in the presence of the stimulus which had preceded the presentation of food. In that experiment the response was conditioned before the contingency between the stimulus and food was set up. In a later experiment (2), a

J. exp. anal. Behav. 1958, 1 (1). 
stimulus preceded food before the lever was introduced. The rat was then reinforced for 10 responses to the lever and, subsequently, on a fixed-interval schedule for 1 hour. When the stimulus was then introduced during extinction, it increased the rate of responding as in the previous experiment.

A similar experiment differing in several significant features has been performed with pigeons. In a first stage a food magazine is operated on an intermittent schedule in the presence of one stimulus but never in the presence of another. In a second stage the pigeon is conditioned to peck a key for food, with neither stimulus present, and a substantial rate is built up with intermittent reinforcement. In the final stage the response is extinguished while the two stimuli are alternately presented. Under these conditions the stimuli were found to exert a differential control over the rate.

\section{APPARATUS AND PROCEDURE}

All inside surfaces of a standard experimental box with inside dimensions of 11 by 10 by 13 inches high were painted a uniform flat white. The space was illuminated by a ceiling light which could be white, red, or green. The magazine presented food for 5 seconds at each operation; a supply of water was present.

Stage 1

Four naive male pigeons were reduced to $80 \%$ of their free-feeding body weight. Each bird was then placed in the box for daily sessions of 144 minutes each. The light in the box was alternately red and green for periods which varied between 6 and 300 seconds, with a mean of 120 seconds. In the presence of one color (red with two pigeons, green with two) the food magazine was operated (without respect to the bird's behavior) on a 1-minute, variable-interval schedule. In the presence of the other color the magazine was never operated. There was no explicit period of "magazine training," but by the second daily session all the birds ate from the magazine whenever it appeared. There were 20 such experimental sessions for each bird.

\section{Stage 2}

A key consisting of a hinged plastic plate behind a 1-inch circular opening at head height in one wall of the box was illuminated only by the ceiling light. In white light all birds were conditioned to peck the key and reinforced 72 times a day for 2 days. They were then reinforced on a 1-minute, variable-interval schedule for 6 days, receiving 72 reinforcements per session. All birds developed stable rates typical of the VI performance in the first session.

\section{Stage 3}

The key was present but responses were never reinforced. (No food was received for any reason.) The illumination in the box was alternately red and green following the schedule of Stage 1. Responses to the key were recorded in the two extinction curves, one showing responses in the red light, the other those in green. Thus, a small segment of one curve was recorded, then a small segment of the other, and so on. The recorder not in use was stopped. A single process of extinction was thus recorded in two curves showing alternating performances in red and green lights. 


\section{RESULTS}

Figure 1 shows the extinction curves obtained in red and green lights for the two pigeons which received food in red light, and Fig. 2 for the two pigeons which received food in green light at Stage 1. In all cases the extinction session began with the red light present.

Bird 1 (Fig. 1) begins to respond almost immediately (at a) in spite of the fact that heretofore the operant has been reinforced only in white light. At $b$ the light was changed to green, and the small segment ending at $\mathbf{c}$ was then recorded. The performance then continues, beginning at $\underline{b}$, and so on, throughout the session. The initial rates of responding in the presence of the two colors are of the same order, but are less than half of the rate on the VI 1 in white light. Beginning at d, responding almost ceases in green light, although it continues at a substantial though irregular rate in red light for at least 2 hours. The experiment was stopped at the end of the records. The total numbers of responses emitted in the red and green lights are in the ratio of 3.3 to 1 .

Bird 2 in Fig. 1 shows a more marked effect of changing from the white light prevailing under VI 1. Both rates begin low but subsequently rise. Here again, responding in the green light drops essentially to zero while there is still considerable activity in red light. The ratio of responses is here about 2 to 1 in favor of red light.

The curves for the two birds receiving food in green light at Stage 1 are shown in Fig. 2. Both birds extinguished fairly rapidly. Bird 3 continues to respond in green light at a high rate for approximately 30 minutes, although responding in

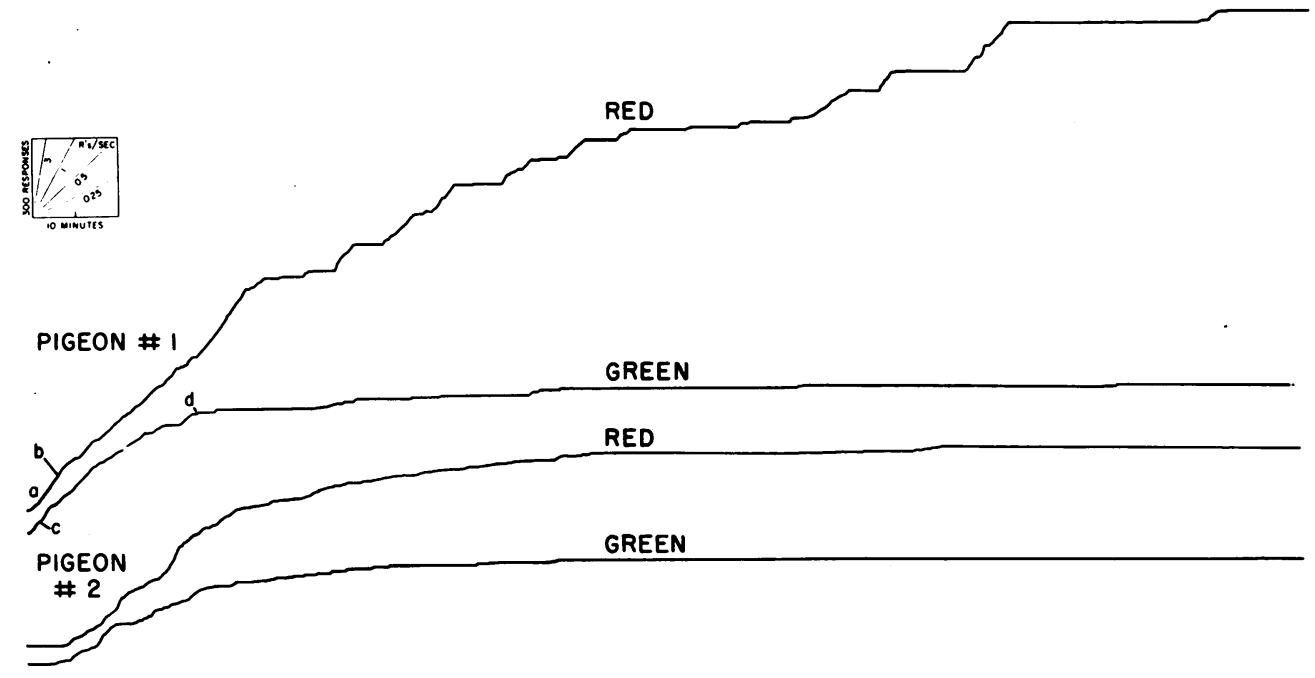

Fig. 1. Performance during extinction recorded separately according to the presence of red and green lights, which alternated randomly. Previously, the pigeons had received food (without respect to behavior) in red light on a 1-minute, variable-interval schedule, but never in the presence of green light. 


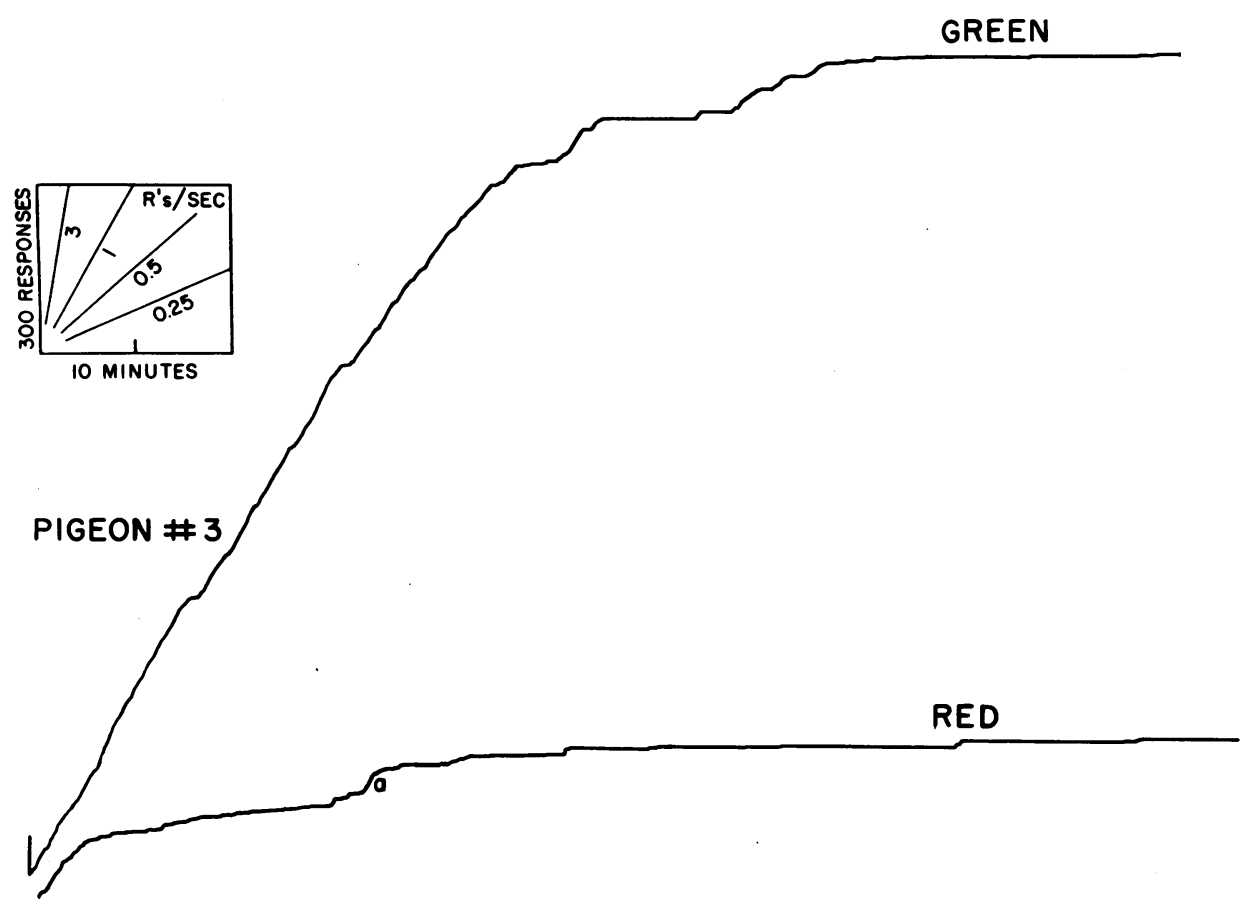

GREEN

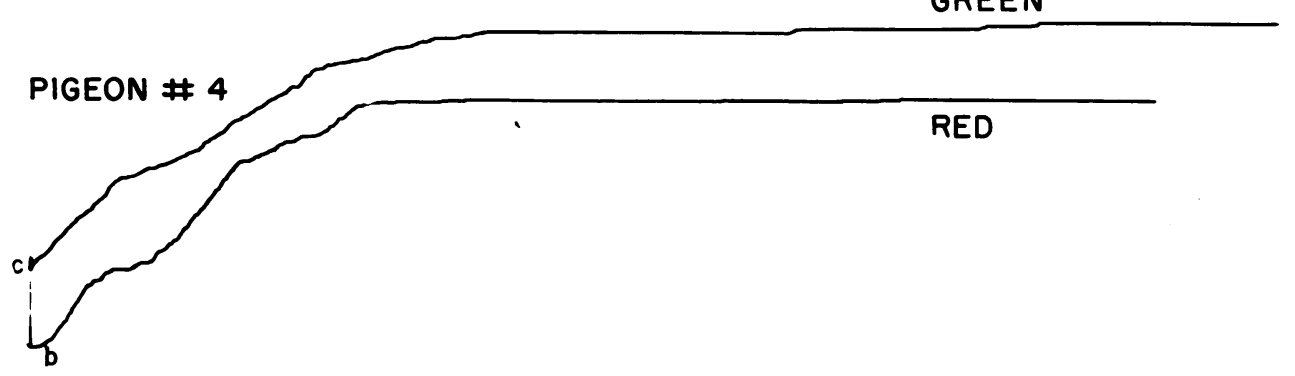

Fig. 2. Performance during extinction recorded separately according to the presence of red and green lights, which alternated randomly. Previously, the pigeons had received food (without respect to behavior) in green light on a 1-minute, variable-interval schedule, but never in the presence of red light. 
red light drops to a low value after about 3 minutes, except for a slight burst at a. The ratio of responses in favor of green (the color present during the reception of food at Stage 1) is 4.5 to 1 .

Bird 4 showed no effect of Stage 1 during the first session of extinction. The session begins at $b$ (Fig. 2) with a slight acceleration in the rate, which decreases slightly when the light is changed to green (beginning at $\mathrm{c}$ ). There are at least two periods of responding in the red light when the rates are considerably more rapid than in the green light, although responding continues for a slightly longer time in the presence of the green light. The numbers of responses in red and green lights are about the same for the session. When this bird was put in the apparatus on a second day, however, it emitted 110 responses in green light (the color previously correlated with food) and 5 in red. (All the other birds also responded more to the food-correlated color during a second extinction session.)

\section{CONCLUSION}

In evaluating these four pairs of extinction curves, it should be noted that the design of the experiment favors a contrary result. The repeated presentation of food to a hungry pigeon usually sets up "superstitious" behavior (4); and during the 20 sessions of Stage 1 , incidental responses were probably reinforced under the discriminative control of the light correlated with food. In extinction at Stage 3 such behavior would compete with the response to the key. The bird would have more time to respond in the presence of the light not correlated with food. The pairs of curves may, therefore, inadequately indicate the extent to which a given light has acquired control over an operant subsequently conditioned.

The experiment confirms Estes' finding that the contingency between $\mathrm{SD}$ and $\mathrm{rft}$ is sufficient to give $S D$ some control over a response subsequently conditioned with rft. In a standard experiment on discrimination, the same temporal correlation may be partly responsible for the ultimate stimulus control. It should be noted, however, that the pairs of extinction curves in the figures show much less control than would have been obtained if Stages 1 and 2 had been combined-- if the response had previously been reinforced in one light and extinguished in the other.

\section{REFERENCES}

1. Estes, W.K. Discriminative conditioning. I. A discriminative property of conditioned anticipation. J. exp. P 8 y c h ol., 1943, 32, 150-155.

2. Estes, W.K. Discriminative conditioning. II. Effects of a Pavlovian conditioned stimulus upon a subsequently established operant response. J . exp. P 8 y c hol ., 1948, 38, 173177.

3. Guttman, N. The pigeon and the spectrum and other perplexities. P sy chol. Rep., 1956, 2, 449-460.

4. Skinner, B. F. 'Superstition' in the pigeon. J. exp. P 8 y c hol ., 1948, 38, 168-172.

5. Walker, K.C. The effect of a discriminative stimulus transferred to a previously unassociated response. J . exp. P s y c hol ., 1942, 31, 312-321. 